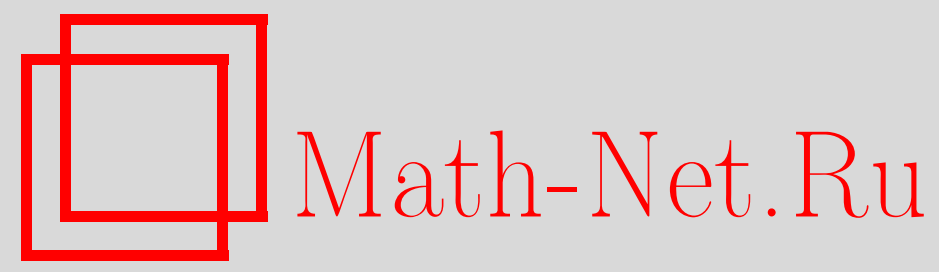

E. Regazzini, V. V. Sazonov, On the central limit problem for partially exchangeable random variables with values in a Hilbert space, Теория вероятн. и ее примен., 1997, том 42, выпуск 4, 796-812

DOI: https://doi.org/10.4213/tvp2597

Использование Общероссийского математического портала Math-Net.Ru подразумевает, что вы прочитали и согласны с пользовательским соглашением http://www . mathnet.ru/rus/agreement

Параметры загрузки:

IP : 54.92 .164 .108

26 апреля 2023 г., 09:59:54

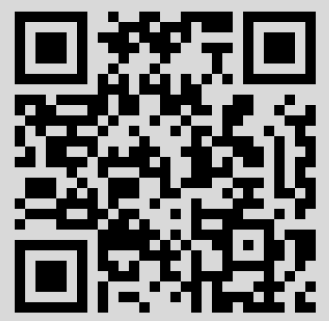


(C) 1997 г. $\quad$ REGAZZINI E. ${ }^{*}$, SAZONOV V. V.**

\title{
ON THE CENTRAL LIMIT PROBLEM FOR PARTIALLY EXCHANGEABLE RANDOM VARIABLES WITH VALUES IN A HILBERT SPACE ${ }^{1)}$
}

\begin{abstract}
В статье рассматривается центральная предельная проблема для перестановочных по столбцам схем серий случайных величин, принимающих значения в гильбертовом пространстве, когда выполнено предположение об условной равномерной асимптотической пренебрегаемости. Доказано, что суммы по строкам могут сходиться по распределению только к перестановочной последовательности случайных величин с распределением, являюшимся смесью безгранично делимых распределений. Даны также условия сходимости к конкретным распределениям, в частности, к смеси гауссовских распределений.
\end{abstract}

Ключевые слова и фразы: центральная предельная проблема, частичная перестановочность, смесь мер, гильбертово пространство.

\section{Introduction}

The main aim of the present paper is to extend a central limit theorem for arrays of partially exchangeable real-valued random variables (r.v.'s), proved in [5], to r.v.'s with values in a real separable Hilbert space $\mathcal{H}$. Even though our proof goes basically along the same lines as in the abovementioned paper [5], it is somewhat simpler and more direct. The approach in [5], as a matter of fact, follows the general approach in proving limit theorems for (partially) exchangeable r.v.'s, namely the reduction via de Finetti's representation to the case of independent r.v.'s. The main tools in the proof, besides the de Finetti representation theorem, are: the Skorokhod theorem on the representation of weakly convergent sequences of probability measures on Polish spaces by almost surely convergent sequences of r.v.'s, and a solution of the central limit problem for independent r.v.'s with values in a Hilbert space. This solution is used here in a somewhat simplified form

*IMQ - Università L. Bocconi, Via Sarfatti 25, 20136 Milano, Italia.

** Математический институт им. В. А. Стеклова РАН, ул. Губкина, 8, 117966 Москва, Россия.

1) Research partially supported by MURST (40\%, 1995, Progetto «Inferenza Statistica: basi probabilistiche e sviluppi metodologici») and by Università Bocconi (fondi di ricerca IMQ, 1995). The results of this paper were obtained while the second author was visiting IAMI-CNR, Milan. 
as compared to what we could find in literature. The conditions for convergence to a Gaussian limit are also presented in a new form. The general setting of Hilbert spaces required some efforts and considerations not arising in the real-valued case.

\section{Preliminaries and notation}

Let $\mathcal{H}$ be a real separable Hilbert space with inner product $(\cdot, \cdot)$ and norm $|\cdot|$. The class of Borel subsets of $\mathcal{H}$ will be denoted by $\mathcal{B}(\mathcal{H})$ and, more generally, $\mathcal{B}(\mathcal{U})$ will stand for the Borel $\sigma$-algebra in any topological space $\mathcal{U}$. Let

$$
\xi=\left\{\xi_{j k}: j=1,2, \ldots ; k=1, \ldots, l\right\}
$$

be an array of partially exchangeable r.v.'s defined on a probability space $(\Omega, \mathcal{F}, \mathbf{P})$ and taking values in $\mathcal{H}$. According to de Finetti [4] that means that, for any finite permutations $\sigma_{1}, \ldots, \sigma_{l}$ of the set $\mathbf{N}$ of all positive integers (i.e., permutations leaving all but a finite number of integers unchanged), the array $\left\{\xi_{\sigma_{k}(j) k}: j=1,2, \ldots ; k=1, \ldots, l\right\}$ has the same distribution as $\xi$. The same argument as used to prove de Finetti's representation theorem for exchangeable r.v.'s shows that partial exchangeability of the elements of $\xi$ is equivalent to the existence of a sub- $\sigma$-algebra $\mathcal{F}^{\prime}$ of $\mathcal{F}$ and of regular conditional distributions $P_{k}(\omega, \cdot)$ on $\mathcal{B}(\mathcal{H})$ given $\mathcal{F}^{\prime}$, such that

$$
\mathbf{P}\left\{\bigcap_{j=1}^{m} \bigcap_{k=1}^{l}\left\{\xi_{j k} \in A_{j k}\right\} \mid \mathcal{F}^{\prime}\right\}=\prod_{j=1}^{m} \prod_{k=1}^{l} P_{k}\left(\omega, A_{j k}\right) \quad \text { (P-a.s.) }
$$

for any Borel subsets of $\mathcal{H}: A_{j k}(j=1, \ldots, m ; k=1, \ldots, l ; m=1,2, \ldots)$. A proof of this statement can be found in [11].

De Finetti's representation (2.1) implies that the elements of $\xi$ are partially exchangeable if and only if there is a probability measure $\rho$ on the product space $\left(\mathcal{P}^{l},(\mathcal{B}(\mathcal{P}))^{l}\right)$ such that

$$
\mathbf{P}\left\{\bigcap_{j=1}^{m} \bigcap_{k=1}^{l}\left\{\xi_{j k} \in A_{j k}\right\}\right\}=\int_{\mathcal{P} l} \prod_{j=1}^{m} \prod_{k=1}^{l} P_{k}\left(A_{j k}\right) \rho\left(d P_{1} \cdots d P_{l}\right)
$$

holds for all $m=1,2, \ldots$, where $\mathcal{P}$ is the Polish space of all probability measures on $\mathcal{B}(\mathcal{H})$ with the topology of weak convergence. The measure $\rho$ is unique and is called the de Finetti measure. Regarding the unicity of $\rho$ see, e.g., Part III in [1].

The above formula can be rewritten in terms of characteristic functions as

$$
\begin{aligned}
\mathbf{E} \exp & \left\{i \sum_{j=1}^{m} \sum_{k=1}^{l}\left(y_{j k}, \xi_{j k}\right)\right\} \\
= & \int_{\mathcal{P}^{\prime}} \prod_{j=1}^{m} \prod_{k=1}^{l} \int_{\mathcal{H}} \exp \left\{i\left(y_{j k}, x_{j k}\right)\right\} P_{k}\left(d x_{j k}\right) \rho\left(d P_{1} \cdots d P_{l}\right) .
\end{aligned}
$$


This follows, e.g., from Corollary 2 of Proposition III-2-1 in [8]. Below we will use a particular case of the above formula, specified for exchangeable sequences. Recall that a sequence of r.v.'s is called exchangeable if its distribution is invariant under all finite permutations of the sequence. From the above it follows that a sequence $\left(\xi_{j}\right)_{j \geqslant 1}$ of $\mathcal{H}$-valued r.v.'s is exchangeable if and only if for any $m=1,2, \ldots$

$$
\mathbf{E} \exp \left\{i \sum_{j=1}^{m}\left(y_{j}, \xi_{j}\right)\right\}=\int_{\mathcal{P}} \prod_{j=1}^{m} \int_{\mathcal{H}} \exp \left\{i\left(y_{j}, x_{j}\right)\right\} p\left(d x_{j}\right) \rho(d p) .
$$

The de Finetti measure $\rho$ in $\left(2.1^{\prime}\right)$ is unique.

Let now

$$
\xi^{(n)}=\left\{\xi_{j k}^{(n)}: j=1,2, \ldots ; k=1, \ldots, k_{n}\right\} \quad(n \in \mathbf{N})
$$

be a sequence of arrays of partially exchangeable $\mathcal{H}$-valued r.v.'s defined on $(\Omega, \mathcal{F}, \mathbf{P})$. Then according to (2.1), there exist a sequence of sub- $\sigma$-algebras $\mathcal{F}^{(n)}$ of $\mathcal{F}$ and a sequence of $k_{n}$-tuples of regular conditional distributions $P_{1 n}(\omega, \cdot), \ldots, P_{k_{n} n}(\omega, \cdot)$ on $\mathcal{B}(\mathcal{H})$ given $\mathcal{F}^{(n)}$ such that

$$
\mathbf{P}\left\{\bigcap_{j=1}^{m} \bigcap_{k=1}^{k_{n}}\left\{\xi_{j k}^{(n)} \in A_{j k}\right\} \mid \mathcal{F}^{(n)}\right\}=\prod_{j=1}^{m} \prod_{k=1}^{k_{n}} P_{k n}\left(\cdot, A_{j k}\right) \quad \text { (P-a.s.) }
$$

for any Borel subsets $A_{j k}$ of $\mathcal{H}\left(j=1, \ldots, m ; k=1, \ldots, k_{n} ; m=1,2, \ldots\right)$.

Like in [5], $\left(\xi^{(n)}\right)_{n \geqslant 1}$ will be called conditionally uniformly asymptotically negligible (conditionally u.a.n.) if for every $\varepsilon>0$

$$
\max _{1 \leqslant k \leqslant k_{n}} P_{k n}\left(\omega, B_{\varepsilon}^{c}\right) \stackrel{\mathbf{P}}{\longrightarrow} 0 \text { as } n \rightarrow+\infty
$$

where $A^{c}$ denotes the complement of $A$ and $B_{\varepsilon}=\{x \in \mathcal{H}:|x|<\varepsilon\}$.

Let $\mathcal{S}$ denote the class of all $S$-operators in $\mathcal{H}$, i.e., linear bounded symmetric nonnegative operators with finite trace. Recall that an $(a, S)$-Gaussian distribution on $\mathcal{B}(\mathcal{H})$ is defined as the Gaussian distribution $Q$ with mean $a=\int_{\mathcal{H}} x Q(d x)$ and covariance operator $S$ in $\mathcal{S}$ :

$$
(S x, y)=\int(x-a, z)(y-a, z) Q(d z) \quad(x, y \in \mathcal{H})
$$

Its characteristic function is

$$
\int_{\mathcal{H}} \exp \{i(x, y)\} Q(d x)=\exp \left\{i(a, y)-\frac{1}{2}(S y, y)\right\} .
$$

This formula gives a bijective mapping from the class of all Gaussian distributions on $\mathcal{B}(\mathcal{H})$, onto $\mathcal{H} \times \mathcal{S}$. In particular there is a one-to-one mapping $\beta$ from the class $\mathcal{G}$ of all centered Gaussian distributions - i.e., $(0, S)$-Gaussian distributions - onto $\mathcal{S}$. The set $\mathcal{G}$, being a closed subset of the Polish space $\mathcal{M}$ of all finite measures on $\mathcal{B}(\mathcal{H})$ with the topology of weak convergence 
(Theorem 1.11 in [10] and, e.g., Theorem 5.4 in Chapter VI of [9]), is also a Polish space in the induced topology. The space $\mathcal{S}$ will be endowed, with the topology transferred from $\mathcal{G}$ by $\beta$ and, consequently, $\mathcal{S}$ will be a Polish space. Note that, from Lemma 5.1, Chapter VI of [9], it follows easily that a sequence $\left(S_{n}\right)_{n \geqslant 1}$ in $\mathcal{S}$ converges to an $S$ in $\mathcal{S}$ (in the topology just introduced) if and oaly if $\left(S_{n} y, y\right) \rightarrow(S y, y)$ for all $y$ in $\mathcal{H}$ and $\left(S_{n}\right)_{n \geqslant 1}$ is compact, i.e.,

$$
\sup _{n} \operatorname{tr} S_{n}<+\infty \text { and } \limsup _{N} \sum_{j=N}^{\infty}\left(S_{n} e_{j}, e_{j}\right)=0
$$

where tr denotes trace and $\left(e_{j}\right)_{j \geqslant 1}$ is an orthonormal basis for $\mathcal{H}$.

Finally, note that $\mathcal{B}(\mathcal{M})$ coincides with the $\sigma$-algebra generated by the family of all functions $\mu(A): \mathcal{M} \rightarrow \mathbf{R}$ as $A$ varies in $\mathcal{B}(\mathcal{H})$ (see [2]). In particular, $\mathcal{M}^{0}=\{\mu \in \mathcal{M}: \mu(\{0\})=0\}$ belongs to $\mathcal{B}(\mathcal{M})$.

\section{Characterization of limiting distributions of sums of partially exchangeable r.v.'s}

Given a sequence $\left(\xi^{(n)}\right)_{n \geqslant 1}$ of arrays of partially exchangeable r.v.'s with $k_{n} \rightarrow+\infty($ as $n \rightarrow+\infty)$, let

$$
Z_{n}=\left(Z_{1 n}, Z_{2 n}, \ldots\right) \quad \text { with } \quad Z_{j n}=\sum_{k=1}^{k_{n}} \xi_{j k}^{(n)} \quad(j=1,2, \ldots) .
$$

The main result in the present section concerns the characterization of the limit of a convergent in distribution $\left(Z_{n}\right)_{n \geqslant 1}$, when $\xi_{j k}^{(n)}$ are conditionally u.a.n. Its proof uses some related results on infinitely divisible distributions in $\mathcal{H}$ and on the central limit problem for independent $\mathcal{H}$-valued r.v.'s.

Proposition 1. (a) A probability measure $\mathbf{P}$ in $\mathcal{P}$ is infinitely divisible if and only if its characteristic function $\widehat{P}(\cdot)=\int_{\mathcal{H}} \exp \{i(x, \cdot)\} \mathbf{P}(d x)$ has the form

$$
\begin{aligned}
\widehat{P}(y) & =\exp \left\{i(a, y)-\frac{1}{2}(S y, y)+\int_{\mathcal{H}^{0}} K(x, y) \mu(d x)\right\} \\
& :=\psi(a, S, \mu ; y) \quad(y \in \mathcal{H}),
\end{aligned}
$$

where $a \in \mathcal{H}, S \in \mathcal{S}, \mathcal{H}^{0}=\mathcal{H} \backslash\{0\}$,

$$
K(x, y):=\left(e^{i(x, y)}-1-\frac{i(x, y)}{1+|x|^{2}}\right) \frac{1+|x|^{2}}{|x|^{2}},
$$

and $\mu \in \mathcal{M}^{0}$. In this representation $a, S$ and $\mu$ are determined uniquely.

(b) Let

$$
\left\{X_{n k}: n=1,2, \ldots ; k=1, \ldots, k_{n}\right\}
$$


be an array of $\mathcal{H}$-valued, rowwise independent r.v.'s defined on $(\Omega, \mathcal{F}, \mathbf{P})$ and satisfying the u.a.n. condition

$$
\lim _{n \rightarrow+\infty} \max _{1 \leqslant k \leqslant k_{n}} \mathbf{P}\left\{X_{n k} \in B_{\varepsilon}^{c}\right\}=0 \quad(\varepsilon>0) .
$$

Fix $\tau>0$ and put

$$
a_{n k}=\int_{B_{\tau}} x P_{n k}(d x)
$$

$P_{n k}$ being the distribution of $X_{n k}$. Furthermore, denote the distribution of $X_{n k}-a_{n k}$ by $\widetilde{P}_{n k}$ and define

$$
a_{n}=\sum_{k=1}^{k_{n}}\left(a_{n k}+\int_{\mathcal{H}} \frac{x}{1+|x|^{2}} \widetilde{P}_{n k}(d x)\right) ;
$$

also define $S_{n k}$ and $S_{n}$ in $\mathcal{S}$ by

$$
\left(S_{n k} y, y\right)=\int_{\mathcal{H}} \frac{(x, y)^{2}}{1+|x|^{2}} \widetilde{P}_{n k}(d x), \quad S_{n}=\sum_{k=1}^{k_{n}} S_{n k} ;
$$

finally define $\mu_{n}$ in $\mathcal{M}$ as

$$
\mu_{n}(A)=\sum_{k=1}^{k_{n}} \int_{A} \frac{|x|^{2}}{1+|x|^{2}} \widetilde{P}_{n k}(d x) \quad(A \in \mathcal{B}(\mathcal{H})) .
$$

Then the sequence $\left(P_{n}\right)_{n \geqslant 1}$, where $P_{n}=P_{n 1} * \cdots * P_{n k_{n}}$ is the distribution of $Z_{n}=\sum_{k=1}^{k_{n}} X_{n k}$, converges weakly to $a \mathbf{P}$ in $\mathcal{P}$ if and only if

(i) $\left|a_{n}-a\right| \rightarrow 0$ for an $a$ in $\mathcal{H}$;

(ii) $\left(S_{n} y, y\right) \rightarrow\left(S^{\prime} y, y\right)$ for an $S^{\prime}$ in $\mathcal{S}$ and for all $y$ in $\mathcal{H}$, and $\left(S_{n}\right)_{n \geqslant 1}$ is compact;

(iii) $\left(\mu_{n}\right)_{n \geqslant 1}$ converges weakly to some $\mu^{\prime}$ in $\mathcal{M}$.

Moreover, if (i)-(iii) are satisfied, then $\left(P_{n}\right)_{n \geqslant 1}$ converges weakly to the infinitely divisible $\mathbf{P}$ in $\mathcal{P}$ with characteristic function

$$
\widehat{P}(y)=\psi(a, S, \mu ; y) \quad(y \in \mathcal{H})
$$

where $S$ is defined by

$$
(S y, y)=\left(S^{\prime} y, y\right)-\int_{\mathcal{H}^{0}} \frac{(x, y)^{2}}{|x|^{2}} \mu(d x)
$$

and

$$
\mu(A)=\mu^{\prime}(A)-\mu^{\prime}(\{0\}) \delta_{0}(A) . \quad(A \in \mathcal{B}(\mathcal{H})),
$$

where $\delta_{0}$ is the probability measure on $\mathcal{B}(\mathcal{H})$ concentrated at the origin.

$\mathrm{R}$ e m a r ks. 1 . It is easy to show (cf. $[6$, p. 393]) that the triple $\left(a, S^{\prime}, \mu^{\prime}\right)$ satisfies the inequality

$$
(S y, y)=\left(S^{\prime} y, y\right)-\int_{\mathcal{H}^{0}} \frac{(x, y)^{2}}{|x|^{2}} \mu(d x) \geqslant 0 \quad(y \in \mathcal{H}) .
$$


Indeed, by definition,

$$
\left(S^{\prime} y, y\right)=\lim _{n} \sum_{k=1}^{k_{n}} \int \frac{(x, y)^{2}}{1+|x|^{2}} d \tilde{P}_{n k}
$$

On the other hand, if $\varepsilon>0$ is such that $\mu^{\prime}\{x:|x|=\varepsilon\}=0$, then the definition of $\mu^{\prime}$ implies

$$
\begin{aligned}
\int_{\mathcal{H}^{0}} \frac{(x, y)^{2}}{|x|^{2}} \mu(d x) & =\int_{B_{c} \backslash\{0\}}+\int_{B_{\varepsilon}^{c}} \\
& \leqslant|y|^{2} \mu^{\prime}\left(B_{\varepsilon} \backslash\{0\}\right)+\lim _{n} \sum_{k=1}^{k_{n}} \int_{B_{\varepsilon}^{c}} \frac{(x, y)^{2}}{1+|x|^{2}} d \widetilde{P}_{n k} .
\end{aligned}
$$

Hence,

$$
(S y, y) \geqslant-|y|^{2} \mu^{\prime}\left(B_{\varepsilon} \backslash\{0\}\right) \rightarrow 0 \text { as } \varepsilon \rightarrow 0 .
$$

Therefore, $S$ does belong to $S$. The transformation mapping $\left(a, S^{\prime}, \mu^{\prime}\right)$ into $(a, S, \mu)$ will be denoted by $T$.

2. In condition (ii) of Proposition 1 the compactness of $\left(S_{n}\right)_{n \geqslant 1}$ can be relaxed by assuming only that

$$
\limsup _{N} \sum_{n=N}^{\infty}\left(S_{n} e_{j}, e_{j}\right)=0 \text { for some basis }\left(e_{j}\right)_{j \geqslant 1} \text { of } \mathcal{H}
$$

since this last relation, together with

$$
\lim _{n}\left(S_{n} y, y\right)=(S y, y)
$$

for every $y$ in $\mathcal{H}$ and for an $S$ in $\mathcal{S}$, implies $\sup _{n} \operatorname{tr} S_{n}=\sup _{n} \mu_{n}(\mathcal{H})<+\infty$; see (5.6) below.

An equivalent form of part (a) of Proposition 1 was proved in [14]; see also [9], [6], [7]. Part (b) was proved in [6] with a different and more complicated definition of $a_{n k}$; see also [7]. With the present definition of $a_{n k}$ it is proved in [12].

We are now in a position to prove

Theorem 1. Let $\left(\xi^{(n)}\right)_{n \geqslant 1}$ be a sequence of conditionally u.a.n. partially exchangeable arrays such that rowwise sums $\left(Z_{n}\right)_{n \geqslant 1}$ converge in distribution. Then there exists a unique probability measure $\nu$ on $(\mathcal{H} \times \mathcal{S} \times$ $\mathcal{M}^{0}, \mathcal{B}\left(\mathcal{H} \times \mathcal{S} \times \mathcal{M}^{0}\right)$ ) such that, for any $m$ in $\mathbf{N}$, the limiting distribution of $Z_{n}^{(m)}=\left(Z_{1 n}, \ldots, Z_{m n}\right)$ has the characteristic function

$$
\varphi_{m}\left(y_{1}, \ldots, y_{m}\right)=\int_{\mathcal{H} \times S \times \mathcal{M}^{\circ}} \prod_{j=1}^{m} \psi\left(a, S, \mu ; y_{j}\right) \nu(d a d S d \mu)
$$

$\left(y_{i} \in \mathcal{H}, i=1, \ldots, m\right)$ 
P r o o f. Let $P_{n}(\omega, \cdot)=P_{1 n}(\omega, \cdot) * \cdots * P_{k_{n} n}(\omega, \cdot)$ where $P_{k n}(\omega, \cdot)$ are the same as in (2.1"). If $P_{n}$ is considered as a mapping from $\Omega$ into $\mathcal{P}$, then $P_{n}$ is measurable and $Q_{n}$ will stand for its distribution.

Claim 1. $\left(Q_{n}\right)_{n \geqslant 1}$ is tight.

To prove this claim, observe that, from $\left(2.1^{\prime \prime}\right)$,

$$
\begin{aligned}
\pi_{n}(A) & :=\mathbf{P}\left\{Z_{1 n} \in A\right\}=\int_{\Omega} P_{n}(\omega, A) \mathbf{P}(d \omega) \\
& =\int_{\mathcal{P}} \lambda(A) Q_{n}(d \lambda) \geqslant x Q_{n}\{\lambda(A)>x\} \quad(x>0)
\end{aligned}
$$

which implies

$$
Q_{n}\{\lambda(A)>x\} \leqslant x^{-1} \pi_{n}(A) \quad(x>0) .
$$

Moreover, from the Prokhorov theorem on equivalence between tightness and relative compactness, $\left(\pi_{n}\right)_{n \geqslant 1}$ is tight since it is weakly convergent. Hence, for any positive integer $m$, there is a compact subset $K_{m}$ of $\mathcal{H}$ such that

$$
\pi_{n}\left(K_{m}^{c}\right) \leqslant m^{-3} \quad(n \in \mathbf{N}) .
$$

Now, given $\varepsilon>0$, define

$$
C_{\varepsilon}=\bigcap_{m=1}^{\infty} E_{m}, \quad \text { where } E_{m}=\left\{\lambda \in \mathcal{P}: \lambda\left(K_{m}^{c}\right) \leqslant\left(\varepsilon_{1} m\right)^{-1}\right\}
$$

and $\varepsilon_{1}=\varepsilon\left(\sum_{1}^{\infty} m^{-2}\right)^{-1}$. Then, if $\left(\lambda_{n}\right)_{n \geqslant 1}$ is a sequence of elements of $E_{m}$ weakly convergent to $\lambda$, from properties of weak convergence it follows that $\varliminf_{n}(G) \geqslant \lambda(G)$ for all open $G$ and, in particular, $\lambda\left(K_{m}^{c}\right) \leqslant\left(\varepsilon_{1} m\right)^{-1}$. Hence, $E_{m}$ is closed for every $m$, which implies that $C_{\varepsilon}$ is closed. Then, since $C_{\varepsilon}$ is obviously tight, from the above-mentioned Prokhorov theorem it follows that $C_{\varepsilon}$ is compact. Now, from (3.5)-(3.6),

$$
Q_{n}\left(\left(C_{\varepsilon}\right)^{c}\right) \leqslant \sum_{m=1}^{\infty} Q_{n}\left(\left(E_{m}\right)^{c}\right) \leqslant \sum_{m=1}^{\infty} \varepsilon_{1} m \pi_{n}\left(\left(K_{m}\right)^{c}\right) \leqslant \sum_{m=1}^{\infty} \varepsilon_{1} m^{-2}=\varepsilon
$$

and this proves Claim 1.

Consider now the sequence of $\mathcal{P}^{\infty}$-valued r.v.'s

$$
\mathbf{P}_{n}(\omega)=\left(P_{n}(\omega, \cdot), P_{1 n}(\omega, \cdot), \ldots, P_{k_{n} n}(\omega, \cdot), \delta_{0}, \delta_{0}, \ldots\right) \quad(n \geqslant 1),
$$

where $\delta_{0}$ here is the random measure identically equal to $\delta_{0}$. Observe that there is an increasing sequence $\left(n^{\prime}\right)$ of positive integers such that

$$
\lim _{n^{\prime} \rightarrow+\infty 1 \leqslant k \leqslant k_{n^{\prime}}} P_{k, n^{\prime}}\left(\omega, B_{\varepsilon}^{c}\right)=0 \quad \text { P-a.s. }(\varepsilon>0) .
$$

To see this, take a sequence $\varepsilon_{m} \downarrow 0$, apply (2.2) to all $\varepsilon=\varepsilon_{m}$, use the fact that every sequence converging in probability contains a subsequence converging almost surely and, finally, apply the diagonal method.

Claim 2. The distributions of the r.v.'s $\mathbf{P}_{n^{\prime}}$ constitute a relatively compact family. 
Indeed, if $f$ is a bounded continuous real-valued function on $\mathcal{H}$, then

$$
\begin{aligned}
& \left|\int_{\mathcal{H}} f(x) P_{k n^{\prime}}(\omega, d x)-\int_{\mathcal{H}} f(x) \delta_{0}(d x)\right| \\
& \quad=\left|\left(\int_{B_{\varepsilon}}+\int_{B_{\varepsilon}^{c}}\right)(f(x)-f(0)) P_{k n^{\prime}}(\omega, d x)\right| \\
& \quad \leqslant \sup _{x \in B_{\varepsilon}}|f(x)-f(0)|+2 \sup |f(x)| P_{k n^{\prime}}\left(\omega, B_{\varepsilon}^{c}\right) .
\end{aligned}
$$

This inequality, by (3.7) and by continuity of $f$, implies that $\left(P_{k n^{\prime}}\right)$ converges weakly to $\delta_{0}\left(\mathbf{P}\right.$-a.s.) as $n^{\prime} \rightarrow+\infty$ and thus, we can conclude that the sequence $\left(Q_{k n^{\prime}}\right)$ of the distributions of r.v.'s $P_{k n^{\prime}}$ converges weakly to the distribution $d_{0}$ concentrated at $\delta_{0}$. These facts and Claim 1 imply componentwise tightness of the sequence of the distributions of r.v.'s $\mathbf{P}_{n^{\prime}}$ and, in view of the Tikhonov theorem on compactness of products of compacts, that sequence itself has to be tight in $\mathcal{P}^{\infty}$. Hence, from the Prokhorov theorem, there is a subsequence of $\left(\mathbb{P}_{n^{\prime}}\right)$ which converges in distribution. Denote such a subsequence by the same symbol $\left(\mathbb{P}_{n^{\prime}}\right)$ and let $\mathbb{Q}$ be its limiting distribution. Since $\mathcal{P}^{\infty}$ is - with respect to the usual product topology - a Polish space, there exists a sequence

$$
\overline{\mathbb{P}}_{n^{\prime}}=\left(\bar{P}_{n^{\prime}}(\bar{\omega}, \cdot), \bar{P}_{1 n^{\prime}}(\bar{\omega}, \cdot), \ldots, \bar{P}_{k_{n^{\prime} n^{\prime}}}(\bar{\omega}, \cdot), \bar{\delta}_{0}, \bar{\delta}_{0}, \ldots\right)
$$

of $\mathcal{P}^{\infty}$-valued r.v.'s, defined on a suitable probability space $(\bar{\Omega}, \overline{\mathcal{F}}, \overline{\mathbf{P}})$, with the same distribution as $\mathbb{P}_{n^{\prime}}$ and converging pointwise to a $\mathcal{P}^{\infty}$-valued r.v. with distribution $\mathbb{Q}$; see 3.1.1 in [13].

Claim 3. $\bar{P}_{n^{\prime}}=\bar{P}_{1 n^{\prime}} * \cdots * \bar{P}_{k_{n^{\prime}} n^{\prime}}(\overline{\mathbf{P}}-a . s)$ for every $n^{\prime}$, and for $a$ subsequence of $n^{\prime}$ (which is denoted again by $n^{\prime}$ )

$$
\lim _{n^{\prime} \rightarrow+\infty} \max _{1 \leqslant k \leqslant k_{n^{\prime}}} \bar{P}_{k n^{\prime}}\left(\bar{\omega},\left(B_{\varepsilon}\right)^{c}\right)=0 \quad(\overline{\mathbf{P}} \text {-a.s. }) \text { for every } \varepsilon>0 .
$$

Firstly, observe that the distributions of

$$
P_{\left(n^{\prime}\right)}=\left(P_{n^{\prime}}, P_{1 n^{\prime}}, \ldots, P_{k_{n^{\prime} n^{\prime}}}\right) \text { and } \bar{P}_{\left(n^{\prime}\right)}=\left(\bar{P}_{n^{\prime}}, \bar{P}_{1 n^{\prime}}, \ldots, \bar{P}_{k_{n^{\prime} n^{\prime}}}\right)
$$

are the same. Then define $\bar{E}_{n^{\prime}}$ to be the subset of $\bar{\Omega}$ on which $\bar{P}_{n^{\prime}}=$ $\bar{P}_{1 n^{\prime}} * \cdots * \bar{P}_{k_{n^{\prime}} n^{\prime}}$. Clearly, $\bar{E}_{n^{\prime}}=\bar{P}_{\left(n^{\prime}\right)}^{-1}\left(F_{n^{\prime}}\right)$ with

$$
F_{n^{\prime}}=\left\{\left(\lambda_{1}, \lambda_{2}, \ldots, \lambda_{k_{n^{\prime}}}\right) \in \mathcal{P}^{k_{n^{\prime}}+1}: \lambda_{1}=\lambda_{2} * \cdots * \lambda_{k_{n^{\prime}}}\right\},
$$

and $F_{n^{\prime}}$ is measurable since it is closed. Hence,

$$
\begin{aligned}
\overline{\mathbf{P}}\left(\bar{E}_{n^{\prime}}\right) & =\overline{\mathbf{P}} \bar{P}_{\left(n^{\prime}\right)}^{-1}\left(F_{n^{\prime}}\right)=\mathbf{P} P_{\left(n^{\prime}\right)}^{-1}\left(F_{n^{\prime}}\right) \\
& =\mathbf{P}\left\{P_{n^{\prime}}=P_{1 n^{\prime}} * \cdots * P_{k_{n^{\prime}} n^{\prime}}\right\}=1 .
\end{aligned}
$$

Furthermore, since $\mathbb{P}_{n^{\prime}}$ and $\overline{\mathbb{P}}_{n^{\prime}}$ have the same distribution,

$$
\max _{1 \leqslant k \leqslant k_{n^{\prime}}} \bar{P}_{k n^{\prime}}\left(\bar{\omega},\left(B_{\varepsilon}\right)^{c}\right) \stackrel{\mathbf{P}}{\longrightarrow} 0 \quad \text { as } n^{\prime} \rightarrow+\infty
$$


and (3.8) is deduced now in exactly the same way as (3.7) was deduced from (2.2).

\section{Claim 4.}

$$
\lim _{n^{\prime} \rightarrow+\infty} \mathbf{E}\left(\prod_{j=1}^{m} \int_{\mathcal{H}} \exp \left\{i\left(y_{j}, x\right)\right\} P_{n^{\prime}}(\cdot, d x)\right)=\overline{\mathbf{E}}\left(\prod_{j=1}^{m} \psi\left(\bar{a}, \bar{S}, \bar{\mu} ; y_{j}\right)\right)
$$

where $\bar{a}, \bar{S}, \bar{\mu}$ are defined as $a, S$ and $\mu$ were defined in Proposition 1 with $\bar{P}_{k n^{\prime}}$ in place of $P_{n^{\prime} k}\left(k=1, \ldots, k_{n^{\prime}}\right)$.

Indeed, from Claim 3 and Proposition 1,

$$
\lim _{n^{\prime} \rightarrow+\infty} \int_{\mathcal{H}} \exp \{i(y, x)\} \bar{P}_{n^{\prime}}(\bar{\omega}, d x)=\psi(\bar{a}(\bar{\omega}), \bar{S}(\bar{\omega}), \bar{\mu}(\bar{\omega}) ; y) \quad(\overline{\mathbf{P}} \text {-a.s. })
$$

and, consequently, by a dominated convergence argument,

$$
\begin{aligned}
\lim _{n^{\prime}} & \int_{\Omega}\left\{\prod_{j=1}^{m} \int_{\mathcal{H}} \exp \left\{i\left(y_{j}, x\right)\right\} P_{n^{\prime}}(\omega, d x)\right\} \mathbf{P}(d \omega) \\
& =\lim _{n^{\prime}} \int_{\bar{\Omega}}\left\{\prod_{j=1}^{m} \int_{\mathcal{H}} \exp \left\{i\left(y_{j}, x\right)\right\} \bar{P}_{n^{\prime}}(\bar{\omega}, d x)\right\} \overline{\mathbf{P}}(d \bar{\omega}) \\
& =\overline{\mathbf{E}}\left(\prod_{j=1}^{m} \psi\left(\bar{a}, \bar{S}, \bar{\mu} ; y_{j}\right)\right) .
\end{aligned}
$$

Now, in order to prove (3.4) it is sufficient to prove that $\bar{a}, \bar{S}$ and $\bar{\mu}$ are r.v.'s with values in $\mathcal{H}, \mathcal{S}$ and $\mathcal{M}^{0}$, respectively, and, subsequently, to apply the change of variable theorem. Since - as it was mentioned in Section 2 $\bar{P}_{k n^{\prime}}(\cdot, A)$ is a real-valued r.v. for each $A$ in $\mathcal{B}(\mathcal{H}), \bar{a}_{n^{\prime} k}, \bar{a}_{n^{\prime}}$ (the same as $a_{n^{\prime} k}$, $a_{n^{\prime}}$ in Proposition 1 with $\bar{P}_{k n^{\prime}}$ in place of $\left.P_{n^{\prime} k}\right)$ are $\mathcal{H}$-valued r.v.'s. Furthermore, $\bar{P}_{k n^{\prime}}(\bar{\omega}, A-x)$, considered as a function of $(\bar{\omega}, x)$, is measurable for each $A$ in $\mathcal{B}(\mathcal{H})$. This can be proved in the same way as the measurability of $m\left(E_{x}\right)$ is usually proved, where $E_{x}$ is the section at $x$ of an $E$ in $\mathcal{B}\left(\mathcal{H}^{2}\right)$ and $m$ is any element of $\mathcal{M}$. Hence, $\bar{P}_{k n^{\prime}}\left(\bar{\omega}, A-\bar{a}_{n^{\prime} k}(\bar{\omega})\right)$ as a function of $\bar{\omega}$ is a real-valued r.v., and this, in its turn, implies the measurability of $\left(\bar{S}_{n^{\prime}} y, y\right)$ and $\bar{\mu}_{n^{\prime}}$ (the same as $\left(S_{n^{\prime}} y, y\right)$ and $\mu_{n^{\prime}}$, respectively, in Proposition 1 with $\bar{P}_{k n^{\prime}}$ in place of $\left.P_{n^{\prime} k}\right)$. Now, from part (b) of Proposition 1 and the construction of $\overline{\mathbb{P}}_{n^{\prime}}$, the sequences $\left(\bar{a}_{n^{\prime}}\right),\left(\left(\bar{S}_{n^{\prime}} y, y\right)\right),\left(\bar{\mu}_{n^{\prime}}\right)$ converge $(\overline{\mathbf{P}}$-a.s. $)$ to $\bar{a},\left(\bar{S}^{\prime} y, y\right), \bar{\mu}^{\prime}$, respectively. Then it follows that these limits are also r.v.'s and, consequently,

$$
\bar{\mu}:=\bar{\mu}^{\prime}-\mu^{\prime}(\{0\}) \delta_{0}, \quad \int_{\mathcal{H}^{\circ}} \frac{(x, y)^{2}}{|x|^{2}} \bar{\mu}(d x),
$$

and

$$
(\bar{S} y, y):=\left(S^{\prime} y, y\right)-\int_{\mathcal{H}^{0}} \frac{(x, y)^{2}}{|x|^{2}} \bar{\mu}(d x)
$$


are r.v.'s. Now it remains to prove only that $\bar{S}$ is a r.v. or, equivalently, that $\bar{G}=\beta^{-1}(\bar{S})$ - where $\beta$ was defined in the Section 2 - is a r.v. To show this, consider the characteristic function

$$
\begin{aligned}
\left(t_{1}, \ldots, t_{r}\right) & \longmapsto \int_{\mathcal{H}} \exp \left\{\sum_{j=1}^{r} t_{j}\left(x, y_{j}\right)\right\} \bar{G}(d x) \\
& =\exp \left\{-\frac{1}{2} \sum_{j, k=1}^{r} t_{j} t_{k}\left(\bar{S} y_{j}, y_{k}\right)\right\},
\end{aligned}
$$

where $y_{j} \in \mathcal{H}, t_{j} \in \mathbf{R}(j=1, \ldots, r)$, and define $\pi_{y}$ to be the projection $x \rightarrow$ $\left(\left(y_{1}, x\right), \ldots,\left(y_{r}, x\right)\right)$ of $\mathcal{H}$ into $\mathbf{R}^{r}$ and let $R_{a b}=\left\{u \in \mathbf{R}^{r}: a_{j}<u_{j} \leqslant b_{j}, j=\right.$ $1, \ldots, r\}$. Then $\bar{G}\left(\pi_{y}^{-1}\left(R_{a b}\right)\right)$ is a r.v., for any $a$ and $b$ in $\mathbf{R}^{r}$. This follows easily from (3.9) and from the inversion formula for characteristic functions of $\mathbf{R}^{r}$-valued r.v.'s. Furthermore, the class of all sets $A$ in $\mathcal{B}\left(\mathbf{R}^{r}\right)$ such that $\bar{G}\left(\pi_{y}^{-1}(A)\right.$ ) (for a fixed $y$ ) is a r.v. forms a monotone class containing the semiring of all rectangles $R_{a b}$. Then that class must contain $\mathcal{B}\left(\mathbf{R}^{r}\right)$. Finally, if $e_{1}, e_{2}, \ldots$ is an orthonormal basis in $\mathcal{H}$, the above argument applied to $y=\widetilde{y}_{r}=\left(e_{1}, \ldots, e_{r}\right), r=1,2, \ldots$, shows that $\bar{G}(A)$ is measurable for all $A$ in the algebra $\mathcal{A}$ of all sets of the form

$$
\pi_{\tilde{y}_{r}}^{-1}(E), \quad E \in \mathcal{B}\left(\mathbf{R}^{r}\right), \quad r=1,2, \ldots
$$

Now, $\mathcal{A}$ generates $\mathcal{B}(\mathcal{H})$, and the class of all $B \subset \mathcal{H}$ such that $\bar{G}(B)$ is measurable is a monotone class. Since this monotone class contains $\mathcal{A}$, then it contains $\mathcal{B}(\mathcal{H})$ as well. The uniqueness of $\nu$ follows from the uniqueness of $\rho$ in $\left(2.1^{\prime}\right)$.

Theorem 1 provides a complete description of the class of all distributions which may represent limiting laws of $\left(Z_{n}\right)_{n \geqslant 1}$, but it does not specify any conditions under which $\left(Z_{n}\right)_{n \geqslant 1}$ converges in distribution. A solution of this problem is given in the next section.

\section{A necessary and sufficient condition for the convergence in distribution of sums of partially exchangeable r.v.'s}

In view of Theorem 1 , conditions for convergence in distribution of $\left(Z_{n}\right)_{n \geqslant 1}$ must be conditions for weak convergence of the law of $Z_{n}$ to a mixture of infinitely divisible distributions. The following theorem specifies a necessary and sufficient condition for that convergence.

Theorem 2. Let $\left(\xi^{(n)}\right)_{n \geqslant 1}$ be a sequence of conditionally u.a.n. partially exchangeable arrays, and let $\nu_{n}$ be the distribution of $\left(a_{n}, S_{n}, \mu_{n}\right)$, where $a_{n}, S_{n}$ and $\mu_{n}$ are defined as in Proposition 1 with $P_{k n}(\omega, \cdot)$ in place of $P_{n k}$. Then the distributions of rowwise sums $Z_{n}$ converge weakly to the limit specified in Theorem 1 by a measure $\nu$, if and only if $\left(\nu_{n}\right)_{n \geqslant 1}$ is relatively compact and $\nu=\nu^{\prime} T^{-1}$ for every accumulation point $\nu^{\prime}$ of $\left(\nu_{n}\right)_{n \geqslant 1}(T$ is defined in Remark 1 above). 
P r o of. To prove necessity, note that if $\left(Z_{n}\right)_{n \geqslant 1}$ converges in law, then, as it was shown in the proof of Theorem 1 , there exists a subsequence $\left(n^{\prime}\right)$ such that $\left(\bar{a}_{n^{\prime}}, \bar{S}_{n^{\prime}}, \bar{\mu}_{n^{\prime}}\right)$ converges almost surely and, hence, in distribution. Then, since $\left(\bar{a}_{n^{\prime}}, \bar{S}_{n^{\prime}}, \bar{\mu}_{n^{\prime}}\right)$ is distributed as $\nu_{n^{\prime}},\left(\nu_{n^{\prime}}\right)$ converges weakly. The same argument applied to any subsequence $\left(Z_{\bar{n}}\right)$ shows that there is a subsequence of $\bar{n}, \bar{n}^{\prime}$ say, such that $\left(\nu_{\bar{n}^{\prime}}\right)$ converges weakly. Hence, $\left(\nu_{n}\right)$ is relatively compact. Now, if $\nu^{\prime}$ is any accumulation point of $\left(\nu_{n}\right)$ and if $\nu_{\bar{n}}$ converges weakly to $\nu^{\prime}$ for some subsequence $(\widetilde{n})$, then the above argument applied to $(\tilde{n})$ implies that there is $\left(\left(\bar{a}_{\tilde{n}^{\prime}}, \bar{S}_{\widetilde{n}^{\prime}}, \bar{\mu}_{\tilde{n}^{\prime}}\right)\right)_{\bar{n}^{\prime}}$ which converges almost surely to a r.v. whose distribution is $\nu^{\prime}$. From this it follows - as in the proof of Theorem 1 - that (3.4) holds for any $m=1,2, \ldots$ with $\nu=\nu^{\prime} T^{-1}$, and this is true for any accumulation point $\nu^{\prime}$ of $\left(\nu_{n}\right)_{n \geqslant 1}$.

Conversely, let $\left(\nu_{n}\right)_{n \geqslant 1}$ be relatively compact and let $\nu=\nu^{\prime} T^{-1}$ for any accumulation point $\nu^{\prime}$ of $\left(\nu_{n}\right)$. For each $n$ in $\mathbf{N}$, define

$$
R_{n}(\omega)=\left(a_{n}(\omega), S_{n}(\omega), \mu_{n}(\omega, \cdot), P_{1 n}(\omega, \cdot), \ldots, P_{k_{n} n}(\omega, \cdot), \delta_{0}, \delta_{0}, \ldots\right) .
$$

Clearly every subsequence of $\left(R_{n}\right)_{n \geqslant 1}$ contains a further subsequence $\left(R_{n^{\prime}}\right)$ which converges in distribution. Furthermore - using the Skorokhod representation theorem - choose a sequence

$$
\bar{R}_{n^{\prime}}=\left(\bar{a}_{n^{\prime}}(\bar{\omega}), \bar{S}_{n^{\prime}}(\bar{\omega}), \bar{\mu}_{n^{\prime}}(\bar{\omega}, \cdot), \bar{P}_{1 n^{\prime}}(\bar{\omega}, \cdot), \ldots, \bar{P}_{k_{n^{\prime}} n^{\prime}}(\bar{\omega}, \cdot), \bar{\delta}_{0}, \bar{\delta}_{0}, \ldots\right)
$$

defined on some probability space $(\bar{\Omega}, \overline{\mathcal{F}}, \overline{\mathbf{P}})$, such that $R_{n^{\prime}}$ and $\bar{R}_{n^{\prime}}$ are identically distributed and $\left(\bar{R}_{n^{\prime}}\right)$ converges a.s. to a r.v. distributed as the limiting distribution of $\left(R_{n^{\prime}}\right)$. A reasoning similar to the proof of Claim 3 in Theorem 1 shows that $\bar{a}_{n^{\prime}}, \bar{S}_{n^{\prime}}$ and $\bar{\mu}_{n^{\prime}}$ coincide a.s. with the quantities defined by (3.1), (3.2) and (3.3), respectively, with $\bar{P}_{k n}$ in place of $P_{n k}$. Also we may assume (choosing a further subsequence if necessary) that $\bar{P}_{k n}$ are u.a.n. a.s. Applying now Proposition 1 we see that $\bar{P}_{n^{\prime}}:=\bar{P}_{1 n^{\prime}} * \cdots * \bar{P}_{k_{n^{\prime}} n^{\prime}}$ weakly converges $(\overline{\mathbf{P}}$-a.s. $)$ to the infinitely divisible distribution, say $\bar{R}$, with parameters $(\bar{a}, \bar{S}, \bar{\mu})=T\left(\bar{a}, \bar{S}^{\prime}, \vec{\mu}^{\prime}\right)$, where $\bar{a}=\lim a_{n^{\prime}}, \bar{S}^{\prime}=\lim \bar{S}_{n^{\prime}}$ and $\vec{\mu}^{\prime}=\lim \vec{\mu}_{n^{\prime}}(\overline{\mathbf{P}}$-a.s. $)$ and $(\bar{a}, \bar{S}, \bar{\mu})$ has distribution $\nu$. Hence for any open $G \subset \mathcal{H}, j=1,2, \ldots$, by the Fatou lemma and the properties of weak convergence

$$
\begin{aligned}
\varliminf_{n^{\prime}} \mathbf{P}\left\{Z_{j n^{\prime}} \in G\right\} & =\frac{\varliminf}{n^{\prime}} \int_{\Omega} P_{n^{\prime}}(\omega, G) \mathbf{P}(d \omega)=\frac{\lim }{n^{\prime}} \int_{\bar{\Omega}} \bar{P}_{n^{\prime}}(\bar{\omega}, G) \overline{\mathbf{P}}(d \bar{\omega}) \\
& \geqslant \int_{\bar{\Omega}} \frac{\varliminf_{n^{\prime}}}{P_{n^{\prime}}}(\bar{\omega}, G) \overline{\mathbf{P}}(d \bar{\omega}) \geqslant \int_{\bar{\Omega}} \bar{R}(\bar{\omega}, G) \overline{\mathbf{P}}(d \bar{\omega}) .
\end{aligned}
$$

- Thus the distributions of r.v.'s $Z_{j n^{\prime}}$ converge weakly to $\int_{\bar{\Omega}} \bar{R}(\bar{\omega}, \cdot) \overline{\mathbf{P}}(d \bar{\omega})$. Consequently the distributions of $Z_{n}=\left(Z_{1 n}, Z_{2 n}, \ldots\right)$ are tight (by Prokhorov's and Tikhonov's theorems). Furthermore assume that, along some subsequence of $\mathbf{N}, Z_{n}$ converges in distribution to a limit. By the above reasoning this subsequence contains a further subsequence $n^{\prime}$ with the properties stated above in the proof of tightness of the distributions of r.v.'s $Z_{n}$. 
In particular for any $m=1,2, \ldots$

$$
\begin{aligned}
\lim _{n^{\prime}} & \mathbf{E} \exp \left\{i \sum_{j=1}^{m}\left(y_{j}, Z_{j n^{\prime}}\right)\right\} \\
= & \lim _{n^{\prime}} \int_{\bar{\Omega}} \prod_{j=1}^{m} \int_{H} \exp \left\{i\left(y_{j}, x\right)\right\} \bar{P}_{n^{\prime}}(\bar{\omega}, d x) \overline{\mathbf{P}}(d \bar{\omega}) \\
= & \int_{\bar{\Omega}} \prod_{j=1}^{m} \psi\left(\bar{a}, \bar{S}, \bar{\mu} ; y_{j}\right) d \overline{\mathbf{P}} \\
= & \int_{\mathcal{H} \times \mathcal{S} \times \mathcal{M}^{0}} \prod_{j=1}^{m} \psi\left(a, S, \mu ; y_{j}\right) \nu(\operatorname{dad} S d \mu) .
\end{aligned}
$$

This shows that the distributions of r.v.'s $Z_{n}$ have only one accumulation point which is characterized by the right-hand side of (4.1) (the same as in $(3.4))$.

\section{Convergence to mixtures of Gaussian distributions}

In the case of arrays of independent r.v.'s, Proposition 1 implies the following criterion of convergence to a Gaussian limit.

Proposition 2. Let

$$
\left\{X_{n k}: n=1,2, \ldots ; k=1, \ldots, k_{n}\right\}
$$

be an array of $\mathcal{H}$-valued, rowwise independent r.v.'s. Then in the notation of Proposition 1:

A. If the sequence $\left(P_{n}\right)_{n \geqslant 1}$ converges weakly, its limit is a Gaussian distribution and the r.v.'s $X_{n k}$ satisfy the u.a.n. condition if and only if

$$
\lim _{n \rightarrow+\infty} \sum_{k=1}^{k_{n}} P_{n k}\left(B_{\varepsilon}^{c}\right)=0 \quad(\varepsilon>0) .
$$

B. The sequence $\left(P_{n}\right)_{n \geqslant 1}$ converges weakly to an $(a, S)$-Gaussian distribution and r.v.'s $X_{n k}$ satisfy the u.a.n. condition if and only if, in addition to (1), the following conditions are satisfied for some $\tau>0$ :

(2) $\lim _{n \rightarrow+\infty}\left|a_{n}^{\prime}-a\right|=0$ with $a_{n}^{\prime}=\sum_{k=1}^{k_{n}} a_{n k}$;

(3) a. $\left(S_{n}^{\prime} y, y\right) \rightarrow(S y, y)$ for all $y$ in $\mathcal{H}$, with $S_{n}^{\prime}=\sum_{k=1}^{k_{n}} S_{n k}^{\prime}$, where

$$
\left(S_{n k}^{\prime} y, y\right)=\int_{B_{r}}(x, y)^{2} P_{n k}(d x)-\left(\int_{B_{r}}(x, y) P_{n k}(d x)\right)^{2}
$$

b. $\left(S_{n}^{\prime}\right)_{n \geqslant 1}$ is compact.

R e m a r k. If $X_{n k}^{\prime}=X_{n k} I_{B_{\tau}}$ (where $I_{B_{\tau}}$ is the indicator function of $B_{\tau}$ ) then $S_{n k}^{\prime}$ is the covariance operator of $X_{n k}^{\prime}$. 
Theorem 6.3, Chapter VI, in [9], is closely related to Proposition 2B, but this theorem provides conditions for convergence of some unspecified shifts of the laws $P_{n}$ to a Gaussian limit rather than of $\left(P_{n}\right)_{n \geqslant 1}$ itself.

$\mathrm{Pr}$ o of of Prop os ition 2. In view of Corollary 6.3, Chapter VI, in [9], to prove part $A$ it suffices to prove that, under the u.a.n. condition, (1) is equivalent to

$$
\lim _{n \rightarrow+\infty} \sum_{k=1}^{k_{n}} \tilde{P}_{n k}\left(B_{\varepsilon}^{c}\right)=0 \text { for every } \varepsilon>0 .
$$

Since

$$
\alpha_{n}:=\sup _{k} \int_{B_{\tau}}|x| P_{n k}(d x) \rightarrow 0 \text { as } n \rightarrow+\infty
$$

(cf. [9, Chapter VI, Lemma 6.1]), then the equivalence at issue follows from the inequalities

$$
\sum_{k} P_{n k}\left(B_{\varepsilon+\alpha_{n}}^{c}\right) \leqslant \sum_{k} \widetilde{P}_{n k}\left(B_{\varepsilon}^{c}\right)=\sum_{k} P_{n k}\left(B_{\varepsilon}^{c}-a_{n k}\right) \leqslant \sum_{k} P_{n k}\left(B_{\varepsilon-\alpha_{n}}^{c}\right)
$$

satisfied when $\alpha_{n}<\varepsilon$.

$\mathrm{N}$ e ces sit y of part B. Conditions (i)-(iii) of Proposition 1 are satisfied with $\mu^{\prime}(\mathcal{H} \backslash\{0\})=0$, so that it suffices to show that they imply (1)-(3). Since (iii) with $\mu^{\prime}(\mathcal{H} \backslash\{0\})=0$ implies

$$
\sum_{k} \widetilde{P}_{n k}\left(B_{\varepsilon}^{c}\right)=\int_{B_{\varepsilon}^{c}} \frac{1+|x|^{2}}{|x|^{2}} \mu_{n}(d x) \longrightarrow 0 \text { as } n \rightarrow+\infty,
$$

then $\left(1^{\prime}\right)$, and hence (1) as well, holds true. Furthermore, for $\delta<\tau$

$$
\begin{aligned}
& \left|\left(S_{n} y, y\right)-\sum_{k} \int_{B_{\tau}}(x, y)^{2} \widetilde{P}_{n k}(d x)\right| \\
& \leqslant|| \sum_{k}\left(\int_{B_{\delta}}+\int_{B_{\tau} \backslash B_{\delta}}\right)(x, y)^{2} \frac{|x|^{2}}{1+|x|^{2}} \widetilde{P}_{n k}(d x) \mid \\
& \quad+\left|\sum_{k} \int_{B_{\tau}^{c}} \frac{(x, y)^{2}}{1+|x|^{2}} \widetilde{P}_{n k}(d x)\right| \\
& \leqslant|y|^{2}\left\{\delta^{2} \sup _{n} \mu_{n}(\mathcal{H})+\tau^{2} \sum_{k} \widetilde{P}_{n k}\left(B_{\delta}^{c}\right)+\sum_{k} \widetilde{P}_{n k}\left(B_{\tau}^{c}\right)\right\} .
\end{aligned}
$$

Together with (ii) and $\left(1^{\prime}\right)$, this gives

$$
\lim _{n \rightarrow+\infty} \sum_{k} \int_{B_{\tau}}(x, y)^{2} \widetilde{P}_{n k}(d x)=(S y, y) \quad(y \in \mathcal{H}) .
$$

On the other hand, if $B_{\tau}(u)=\{x \in \mathcal{H}:|x-u|<\tau\}$ and $\alpha_{n}<\tau$, then

$$
B_{\tau} \Delta B_{\tau}\left(-a_{n k}\right) \subset\left\{x: \tau-\alpha_{n} \leqslant|x| \leqslant \tau+\alpha_{n}\right\}:=B_{n \tau}
$$


and, consequently,

$$
\begin{aligned}
& \left|\sum_{k}\left(\int_{B_{\tau}}-\int_{B_{\tau}\left(-a_{n k}\right)}\right)(x, y)^{2} \widetilde{P}_{n k}(d x)\right| \\
& \quad \leqslant \sum_{k} \int_{B_{n \tau}}(x, y)^{2} \widetilde{P}_{n k}(d x) \\
& \quad \leqslant|y|^{2}\left(\tau+\alpha_{n}\right)^{2} \sum_{k} \widetilde{P}_{n k}\left(B_{\tau-\alpha_{n}}^{c}\right) \longrightarrow 0 \text { as } n \rightarrow+\infty .
\end{aligned}
$$

From (5.1)-(5.2) it follows that

$$
\lim _{n \rightarrow+\infty} \sum_{k} \int_{B_{\tau}}\left(x-a_{n k}, y\right)^{2} P_{n k}(d x)=(S y, y) \quad(y \in \mathcal{H})
$$

Finally

$$
\begin{aligned}
\sum_{k} & \int_{B_{\tau}}\left(x-a_{n k}, y\right)^{2} P_{n k}(d x) \\
= & \sum_{k}\left[\int_{B_{\tau}}(x, y)^{2} P_{n k}(d x)-\left(\int_{B_{\tau}}(x, y) P_{n k}(d x)\right)^{2}\right] \\
& -\sum_{k}\left(a_{n k}, y\right)^{2} \int_{B_{\tau}^{c}} P_{n k}(d x)
\end{aligned}
$$

and, since the last term on the right side converges to 0 by (1) and by the inequality $\left|a_{n k}\right| \leqslant \sup _{n} \alpha_{n}<+\infty$, (3)a holds true.

(3)b can be deduced from (ii) in a similar way and, therefore, it remains to prove (2). To this aim, note that

$$
\begin{aligned}
\sum_{k} \int_{\mathcal{H}} \frac{x}{1+|x|^{2}} \widetilde{P}_{n k}(d x)= & \sum_{k} \int_{B_{\tau}} x \widetilde{P}_{n k}(d x)-\int_{B_{\tau}} x \mu_{n}(d x) \\
& +\int_{B_{\tau}^{c}} \frac{x}{|x|^{2}} \mu_{n}(d x)
\end{aligned}
$$

such that, by (iii) and by the equality $\mu^{\prime}(\mathcal{H} \backslash\{0\})=0$, the two last integrals on the right side converge to 0 as $n \rightarrow+\infty$. Moreover, if $\alpha_{n}<\tau$, then likewise in (5.2),

$$
\begin{aligned}
\left|\sum_{k} \int_{B_{\tau}} x \widetilde{P}_{n k}(d x)\right| & =\left|\sum_{k}\left(\int_{B_{\tau}}-\int_{B_{\tau}\left(-a_{n k}\right)}\right) x \widetilde{P}_{n k}(d x)\right| \\
& \leqslant\left(\tau+\alpha_{n}\right) \sum_{k} \widetilde{P}_{n k}\left(B_{\tau-\alpha_{n}}^{c}\right) \rightarrow 0 \quad(n \rightarrow+\infty)
\end{aligned}
$$

and (2) follows.

$\mathrm{S}$ u f f i c i e n c y of p a r t B. It suffices to prove that (1)-(3) imply conditions (i)-(iii) of Proposition 1 with $\mu^{\prime}(\mathcal{H} \backslash\{0\})=0$. Obviously, from 
(1) it follows that the r.v.'s $X_{n k}$ satisfy the u.a.n. condition. Hence, $\left(1^{\prime}\right)$ is also true and, for any $\varepsilon>0$,

$$
\mu_{n}\left(B_{\varepsilon}^{c}\right) \leqslant \sum_{k} \widetilde{P}_{n k}\left(B_{\varepsilon}^{c}\right) \rightarrow 0 \text { as } n \rightarrow+\infty .
$$

Furthermore, by reversing the reasoning in the necessity part and using (1), one can show that (ii) follows from (3). Observe now that (ii) implies

$$
\mu_{n}(\mathcal{H}) \rightarrow \operatorname{tr} S \quad(n \rightarrow+\infty)
$$

Indeed, for any $N$ in $\mathbf{N}$,

$$
\begin{aligned}
\left|\mu_{n}(\mathcal{H})-\operatorname{tr} S\right| & =\left|\sum_{j=1}^{\infty}\left(S_{n} e_{j}, e_{j}\right)-\sum_{j=1}^{\infty}\left(S e_{j}, e_{j}\right)\right| \\
& \leqslant \sum_{j=1}^{N-1}\left|\left(S_{n} e_{j}, e_{j}\right)-\left(S e_{j}, e_{j}\right)\right|+\sum_{j=N}^{\infty}\left(\left(S_{n} e_{j}, e_{j}\right)+\left(S e_{j}, e_{j}\right)\right)
\end{aligned}
$$

and, if $N$ is chosen large enough, the second sum on the right side can be made arbitrarily small, while the first sum, for a fixed $N$, can be made arbitrarily small as $n \rightarrow+\infty$. Now, from (5.4) and (5.5) it follows easily that (iii) holds with $\mu^{\prime}(\mathcal{H} \backslash\{0\})=0$. Finally, in the necessity part, it was shown that $\left(1^{\prime}\right)$ and (iii) with $\mu^{\prime}(\mathcal{H} \backslash\{0\})=0$ imply (5.3), and thus (i) follows from (2).

Now we can give conditions for the weak convergence of the distributions of the r.v.'s $Z_{n}$ - defined at the beginning of Section 3 - to a Gaussian mixture. A probability measure $R$ on $\mathcal{B}\left(\mathcal{H}^{\infty}\right)$ is said to be a Gaussian mixture if there exists a probability $\gamma$ on $\mathcal{B}(\mathcal{H} \times \mathcal{S})$ such that

$$
\begin{gathered}
\int_{\mathcal{H}^{\infty}} \exp \left\{i \sum_{j=1}^{m}\left(x_{j}, y_{j}\right)\right\} R(d x) \\
=\int_{\mathcal{H} \times \mathcal{S}} \prod_{j=1}^{m} \exp \left\{i\left(a, y_{k}\right)-\frac{1}{2}\left(S y_{j}, y_{j}\right)\right\} \gamma(d a d S) \\
\left(m \in \mathbf{N}, y_{1}, \ldots, y_{m} \in \mathcal{H}\right) .
\end{gathered}
$$

Below $\gamma_{n}$ denotes the distribution of $\left(a_{n}^{\prime}, S_{n}^{\prime}\right)$, where $a_{n}^{\prime}$ and $S_{n}^{\prime}$ are the same as in Proposition 2 with $P_{k n}(\omega, \cdot)$ in place of $P_{n k}$, and $P_{k n}(\omega, \cdot)$ are from representation $\left(2.1^{\prime \prime}\right)$.

Theorem 3. Let $\left(\xi^{(n)}\right)_{n \geqslant 1}$ be a sequence of partially exchangeable arrays. Then

A. If the distributions of the r.v.'s $Z_{n}$ converge weakly, their limit is a Gaussian mixture and the arrays $\left(\xi^{(n)}\right)_{n \geqslant 1}$ are conditionally u.a.n. (i.e., 
(2.2) holds), if and only if

$$
\sum_{k=1}^{k_{n}} P_{k n}\left(\omega, B_{\varepsilon}^{c}\right) \stackrel{\mathbf{P}}{\longrightarrow} 0 \quad(\varepsilon>0, n \rightarrow+\infty) ;
$$

B. The distributions of r.v.'s $Z_{n}$ converge weakly to the Gaussian mixture with de Finetti's measure $\gamma$ and (2.2) holds if and only if (5.7) is satisfied and $\gamma_{n}$ converges weakly to $\gamma$.

$\mathrm{P}$ r o of. $\mathrm{N}$ e ce s s i t y. Assume that the distributions of r.v.'s $Z_{n}$ converge to a Gaussian mixture and that (2.2) holds. Then, like in the proof of Theorem 1, and according to the notation introduced there, there is an increasing subsequence $\left(n^{\prime}\right)$ of $\mathbf{N}$ such that $\left(\bar{\mu}_{n^{\prime}}\right)$ converges weakly $\left(\overline{\mathbf{P}}\right.$-a.s.) to a limit $\vec{\mu}^{\prime}$ and $\bar{P}_{k n^{\prime}}$ satisfy (3.8). Moreover, the distribution of $\vec{\mu}^{\prime}-\vec{\mu}^{\prime}(\{0\}) \delta_{0}$ is the $\mu$-component of the measure $\nu$ in (3.4). Hence, from the assumption that the limit is a Gaussian mixture and by the unicity of de Finetti's measure:

$$
\bar{\mu}^{\prime}(\mathcal{H})-\bar{\mu}^{\prime}(\{0\})=0 \quad\left(\overline{\mathbf{P}}_{\text {-a.s. }}\right) .
$$

Then, for any $\varepsilon>0$,

$$
\begin{aligned}
\sum_{k=1}^{k_{n^{\prime}}} \tilde{\bar{P}}_{k n^{\prime}}\left(\bar{\omega}, B_{\varepsilon}^{c}\right) & \leqslant \frac{1+\varepsilon^{2}}{\varepsilon^{2}} \sum_{k=1}^{k_{n^{\prime}}} \int_{B_{\varepsilon}^{c}} \frac{|x|^{2}}{1+|x|^{2}} \widetilde{\bar{P}}_{k n^{\prime}}(\bar{\omega}, d x) \\
& =\frac{1+\varepsilon^{2}}{\varepsilon^{2}} \bar{\mu}_{n^{\prime}}\left(B_{\varepsilon}^{c}\right) \rightarrow 0 \quad(\overline{\mathbf{P}} \text {-a.s., } n \rightarrow+\infty)
\end{aligned}
$$

and (see the proof of the equivalence of (1) and (1') in Proposition 2)

$$
\lim _{n \rightarrow+\infty} \sum_{k=1}^{k_{n^{\prime}}} \bar{P}_{k n^{\prime}}\left(\bar{\omega}, B_{\varepsilon}^{c}\right)=0 \quad(\overline{\mathbf{P}} \text {-a.s. }) .
$$

Consequently, $\sum_{k=1}^{k_{n^{\prime}}} \bar{P}_{k n^{\prime}}\left(\bar{\omega}, B_{\varepsilon}^{c}\right)$ converges to 0 in distribution, as $n \rightarrow+\infty$, for every $\varepsilon>0$. Since in the above reasoning we could start with any increasing subsequence of $\mathbf{N}$, instead of $\mathbf{N}$ itself, (5.7) holds true.

Assume now that the distributions of r.v.'s $Z_{n}$ converge weakly to the Gaussian mixture characterized by $\gamma$. Then, each subsequence of $\mathbf{N}$ includes a further subsequence $\left(n^{\prime}\right)$ such that, in our usual notation, $\left(\vec{a}_{n^{\prime}}^{\prime}, \bar{S}_{n^{\prime}}^{\prime}\right)$ converges $\left(\overline{\mathbf{P}}_{\text {-a.s. }}\right)$ and (5.8) is satisfied. This follows from the proof of Theorem 1 - employing Proposition 2 instead of Proposition 1 - and from the proof of (5.8). Now, proceeding as in the proof of Theorem 2, we conclude that $\gamma_{n}$ converges weakly to $\gamma$.

$\mathrm{S}$ u f $\mathrm{f}$ i c i e $\mathrm{n} \mathrm{c}$ y. To prove sufficiency in part $\mathrm{B}$, consider the sequence

$$
\left(\left(a_{n}^{\prime}(\omega), S_{n}^{\prime}(\omega), P_{1 n}(\omega, \cdot), \ldots, P_{k_{n} n}(\omega, \cdot), \delta_{0}, \delta_{0}, \ldots\right)\right)_{n \geqslant 1} .
$$

An argument similar to the one used to prove sufficiency in Theorem 2 shows that each subsequence of $\mathbf{N}$ contains a further subsequence $\left(n^{\prime}\right)$ along which 
(5.8) holds, $\lim _{n^{\prime}}, \bar{a}_{n^{\prime}}^{\prime}=\bar{a}, \lim _{n^{\prime}} \bar{S}_{n^{\prime}}^{\prime}=\bar{S}$ exis.t $(\overline{\mathbf{P}}$-a.s. $)$, and $\bar{P}_{n^{\prime}}$ converges weakly to the $(\bar{a}, \bar{S})$-Gaussian distribution $(\overline{\mathbf{P}}$-a.s. $)$. The rest of the proof goes as in Theorem 2.

Finally, the sufficiency part in A can be proved by the same argument applied to the sequence

$$
\left(\left(P_{1 n}(\omega, \cdot), \ldots, P_{k_{n} n}(\omega, \cdot), \delta_{0}, \delta_{0}, \ldots\right)\right)_{n \geqslant 1} .
$$

\section{REFERENCES}

1. Aldous $D$. J. Exchangeability and related topics. - In: École d'Été de Probabilités de Saint-Flour XIII-1983./Ed. by P. L. Hennequin et al. (Lect. Notes Math. Vol. 1117.) Berlin: Springer-Verlag, 1985, p. 2-198.

2. Berk R. H., Savage I. R. Dirichlet processes produce discrete measures: an elementary proof. - In: Contributions to Statistics./Ed. by J. Jurečkova Dordrecht: Reidel, 1979, p. 25-31.

3. Billingsley $P$. Convergence of Probability Measures. New York: Wiley, 1968.

4. de Finetti $B$. Sur la condition d'équivalence partielle. (Actualités Scientifiques et Industrielles, No. 739.) Paris: Hermann, 1938.

5. Fortini $S$., Ladelli $L$., Regazzini $E$. A central limit problem for partially exchangeable random variables. - Теория вероятн. и ее примен., 1996, т. 41, в. 2, с. 353-379.

6. Gihman I. I., Skorohod A. V. The theory of stochastic processes I. Berlin: SpringerVerlag, 1979.

7. Laha R. G., Rohatgi V. K. Probability Theory. New York: Wiley, 1979.

8. Neveu J. Bases mathématiques du calcul des probabilités. Paris: Masson, 1980.

9. Parthasarathy K. R. Probability Measures on Metric Spaces. San Diego: Academic Press, 1967.

10. Прохоров Ю. В. Сходимость случайных процессов и предельные теоремы теории вероятностей. - Теория вероятн. и ее примен., 1956, т. 1, в. 2, с. 177-238.

11. Regazzini $E$. Coherence, exchangeability and statistical models (de Finetti's stances «revisited»). - - In: Atti del Convegno «Sviluppi metodologici nei diversi approcci all'inferenza statistica». Vol. 2. Bologna: Pitagora, 1991, p. 101-137.

12. Sazonov $V$. V. A simplified solution of the central limit problem in Hilbert space. Technical report № 504, Center for Stochastic Processes, University of North Carolina at Chapel Hill, 1997.

13. Скороход A. В. Предельные теоремы для случайных процессов. - Теория вероятн. и ее примен., 1956, т. 1, в. 3, с. 289-319.

14. Varadhan $S$. R. S. Limit theorems for sums of independent random variables with values in a Hilbert space: - Sankhyā, Ser. A, 1962, v. 24, p. 213-238. 